\title{
Structural and magnetic properties of the (001) and (111) surfaces of the half-metal NiMnSb
}

\author{
M Ležaić†, I Galanakisț, G Bihlmayer $\dagger$ and S Blügel $\dagger$ \\ † Institut für Festkörperforschung, Forschungszentrum Jülich, D-52425 Jülich, \\ Germany \\ ¥ Institut of Microelectronics, NCSR "Demokritos", 15310 Aghia Paraskevi, \\ Athens, Greece \\ E-mail: I.Galanakis@fz-juelich.de
}

\begin{abstract}
Using the full potential linearised augmented planewave method we study the electronic and magnetic properties of the (001) and (111) surfaces of the half-metallic Heusler alloy NiMnSb from first-principles. We take into account all possible surface terminations including relaxations of these surfaces. Special attention is paid to the spin-polarization at the Fermi level which governs the spin-injection from such a metal into a semiconductor. In general, these surfaces lose the half-metallic character of the bulk NiMnSb, but for the (111) surfaces this loss is more pronounced. Although structural optimization does not change these features qualitatively, specifically for the (111) surfaces relaxations can compensate much of the spin-polarization at the Fermi surface that has been lost upon formation of the surface.
\end{abstract}

PACS numbers: 75.47.Np, 73.20.At, 71.20.Lp

Submitted to: Journal of Physics: Condensed Matter 


\section{Introduction}

During the last decade the emergence of the fields of magnetoelectronics and spintronics has given birth to a new series of challenges in materials science [1 2]. A central problem remains the spin-injection from a ferromagnet into a semiconductor 3. Its successful realization would lead to the creation of a series of novel devices such as spin-filters [4], tunnel junctions [5] or GMR devices for spin injection [6]. The use of half-metallic ferromagnets as electrodes was proposed to maximize the efficiency of such spintronic devices. These compounds are ferromagnetic metals with a band gap at the Fermi level $\left(E_{\mathrm{F}}\right)$ in the minority spin channel leading to $100 \%$ spin-polarization at $E_{\mathrm{F}}$. Thus, in principle, during the spin-injection process only majority-spin electrons would be injected in the semiconductor.

The family of half-metallic systems which has attracted most of the attention are the half-Heusler alloys and especially NiMnSb. These compounds of the general formula XYZ crystallize in the $C 1_{b}$ structure, which consists of $4 \mathrm{fcc}$ sublattices occupied by the three atoms X, Y and Z and a vacant site [7. In 1983 de Groot and his collaborators were the first to predict the half-metallic character of $\mathrm{NiMnSb}$ on the basis of first-principles calculations [8]. Thereafter, several ab-initio calculations on NiMnSb reproduced these results [9], and Galanakis et al. showed that the gap arises from the hybridization between the $d$ orbitals of the Ni and Mn atoms [10]. This explanation was confirmed also by the work of Nanda and Dasgupta 11. Several other studies were performed on the stability of the minority-spin band-gap which was found to be stable under hydrostatic pressure and tetragonalization 12 or a small disorder 13 but the exchange of the atoms occupying the different sublattices completely destroys the gap 14. Experiments seem to well establish the half-metallicity in the case of NiMnSb single crystals [15, 16] or away from the surface in the case of thick films [17.

Recently, high quality films of NiMnSb alloys have been also grown 18, 19, 20, but they were found to be not half-metallic 21, 22]; a maximum value of $58 \%$ for the spinpolarisation of NiMnSb was obtained by Soulen et al. 21]. These polarisation values are consistent with a small perpendicular magnetoresistance measured for $\mathrm{NiMnSb}$ in a spin-valve structure [23, and a superconducting and a magnetoresistive tunnel junction [5]. Ristoiu et al. showed that during the growth of the NiMnSb thin films, first $\mathrm{Sb}$ and then $\mathrm{Mn}$ atoms segregate to the surface, which is far from being perfect, thus decreasing the obtained spin-polarization [24. But when they removed the excess of Sb by a flash annealing, they managed to get a nearly stoichiometric ordered alloy surface being terminated by a MnSb layer, which presented a spin-polarization of about $67 \pm 9 \%$ at room temperature 24. Even in the case of perfect surfaces, these would not be half-metallic due to surface states [25], non-quasiparticle states [26] and finite-temperature effects [27. There is evidence that around $80 \mathrm{~K}$ the films undergo a transition towards a normal metal [28].

First-principles calculations have been also employed to study the surfaces of $\mathrm{NiMnSb}$. Jenkins and King were the first to study by a pseudopotential technique the MnSb terminated (001) surface of NiMnSb and showed that there are two surface states at the Fermi level, which are well localised in the surface layer [29]. They have also shown that there is a small relaxation of the surface with the Mn atoms moving slightly inwards and the Sb outwards and this small relaxation is energetically more favourable than the creation of $\mathrm{Mn}$ or $\mathrm{Sb}$ dimers forming in a $c(2 \times 2)$ reconstruction. Galanakis studied also the (001) surfaces using the full-potential version of the 
Korringa-Kohn-Rostoker Green function method (FSKKR) [25]. He found that the MnSb-terminated surface shows a quite large spin-polarization in agreement with the experiments of Ristoiu et al. [24].

In a study of (111) terminated NiMnSb surfaces, Jenkins investigated the relative stability of stoichiometric surfaces as well as their stability with respect to to other, nonstoichiometric structures [30. The spin moments and surface states of the (111) surfaces of NiMnSb have been studied by Galanakis, who found in all cases very pronounced surface states on the unrelaxed (111) surfaces [31]. These calculations have been obtained within the atomic sphere approximation which can lead to errors for surfaces with respect to the full-potential ones.

In this communication, we present ab-initio calculations of the (001) and (111) surfaces of the half-metallic NiMnSb Heusler alloy. We take into account all possible terminations and study the electronic and magnetic properties of the surfaces and calculate the spin-polarization at the Fermi level. We also investigate the effect of the relaxation of the atomic positions of the atoms near the surface on the electronic and magnetic properties of the surfaces. Section 2 presents the method of the calculation. In section 3 we briefly review the bulk properties of NiMnSb and sections 4 and 5 are devoted to the (001) and (111) surfaces, respectively. Finally in section [6 we resume and conclude.

Prior to presenting our results we should make two important notices. First, based on the experiences from ferromagnets and semiconductors, two effects should be particularly relevant for the surfaces of half-metals: (i) in magnetic systems, the moments of the surface atoms are strongly enhanced due to the missing hybridization with the cut-off neighbours, and (ii) in semiconductors, surface states can appear in the gap such that the surface often becomes metallic. Also this is a consequence of the reduced hybridization, leading to dangling bond states in the gap. Secondly, it should be mentioned that at an interface, the interface states will certainly differ from the surface states studied here. But to a certain extent, the surface states for a given surface orientation will have characteristics also typical for interface states. In principle these states should not affect the magnetoconductance since the wavefunction is orthogonal to all bulk states incident to the surface. But emission or absorption of magnons couples weakly the bulk and surface states and affects the magnetotransport. In real systems the interaction of the surface states with other defect states in the bulk and/or with surface defects makes the surface states conducting and leads to the low spin-polarization values for films derived by Andreev reflection measurements.

\section{Method and structure}

The calculations were performed using density functional theory and the generalized gradient approximation (GGA) as given by Perdew et al. [32. We use the fullpotential linearised augmented planewave (FLAPW) method in film geometry 33, as implemented in the FLEUR program [34]. For the calculations, a planewave cutoff $K_{\max }$ of 3.6 a.u. $^{-1}$ was used. Lattice harmonics with angular momentum $l \leq 8$ were used to expand the charge density and the wavefunctions within the muffin-tin spheres. In the case of the (001) surfaces we used a film consisting of 9 atomic layers of $\mathrm{NiMnSb}$, while for the (111) interfaces 13 layers were used. The two-dimensional Brillouin-zone was sampled with 64 special k-points in the irreducible wedge for the (001) surfaces, and $90 \mathrm{k}$-points in the irreducible wedge for the (111) surfaces. All the calculations were performed at the optimized lattice constant of $\mathrm{NiMnSb}(5.915 \AA)$ which is within 


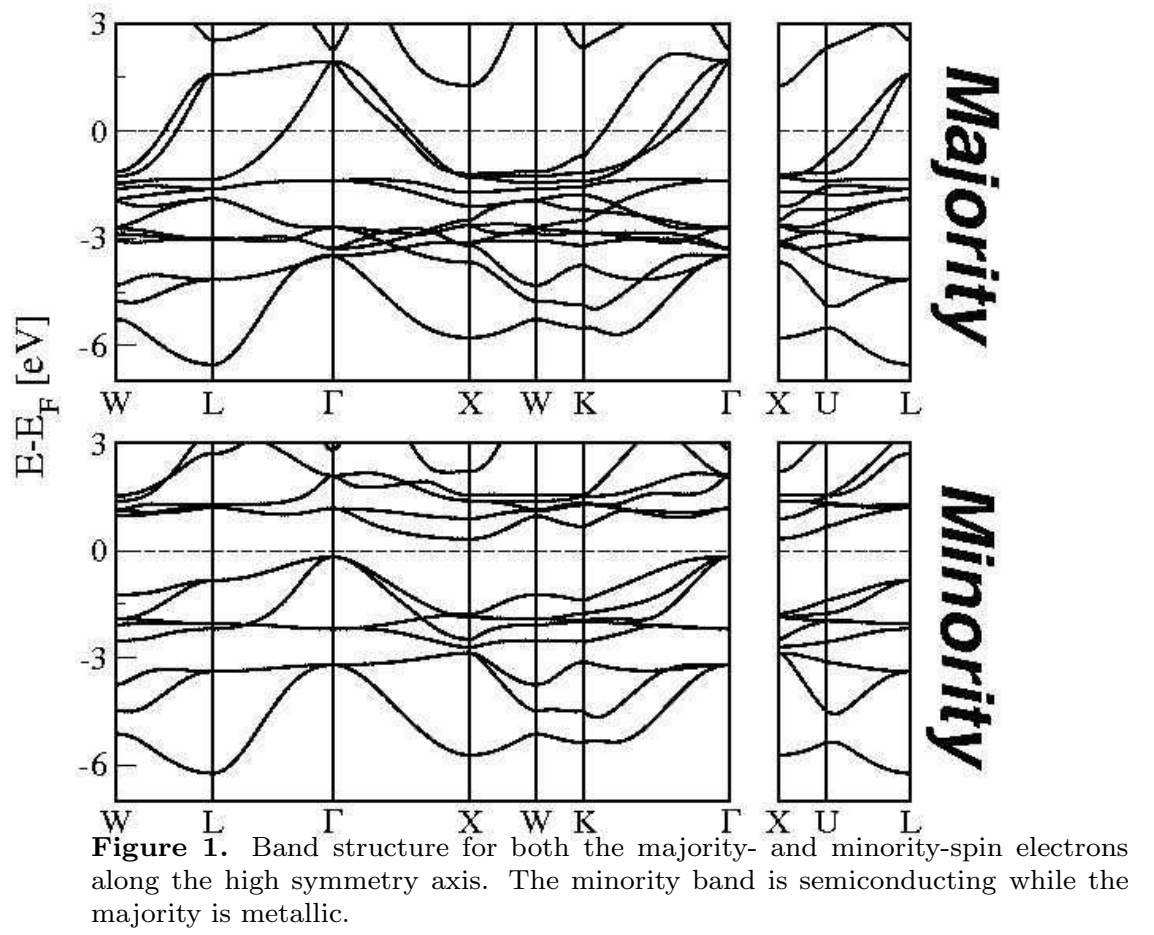

$0.2 \%$ agreement with the experimental value 35. Structural optimization was done by minimizing the forces on the three topmost layers of the film.

There are two different possible terminations in the case of the (001) surfaces, one containing the $\mathrm{Mn}$ and $\mathrm{Sb}$ atoms while the other contains only a $\mathrm{Ni}$ atom [25], 29]. The interlayer distance is one fourth of the bulk lattice constant. In the perpendicular direction the layer occupancy is repeated every fourth layer, since in the $i \pm 2$ layer the atoms have exchanged positions compared to the $i$ layer.

In the case of the (111) surfaces we have more different surface terminations as compared to the (001) ones. Along the [111] direction the alloy consists of alternating closed packed layers containing only one chemical element per layer. For example in the case of a Ni-terminated surface, there are two different possibilities: either to have a Mn subsurface layer or an $\mathrm{Sb}$ one (see [30] or 31] for the different terminations). In total we can identify six different surface terminations.

\section{Bulk properties}

The bulk properties of NiMnSb have been extensively studied during the last years and it is still considered to be a key component in the search for spintronic devices. Thus, in this section we will only briefly discuss its bulk properties. NiMnSb, as predicted by de Groot and collaborators, is a half-metal [8]. To explain this we present in figure 1 the band structure of this compound for both majority- (upper panel) and minority-spin (bottom panel) electrons. The majority-spin bands are characteristic 


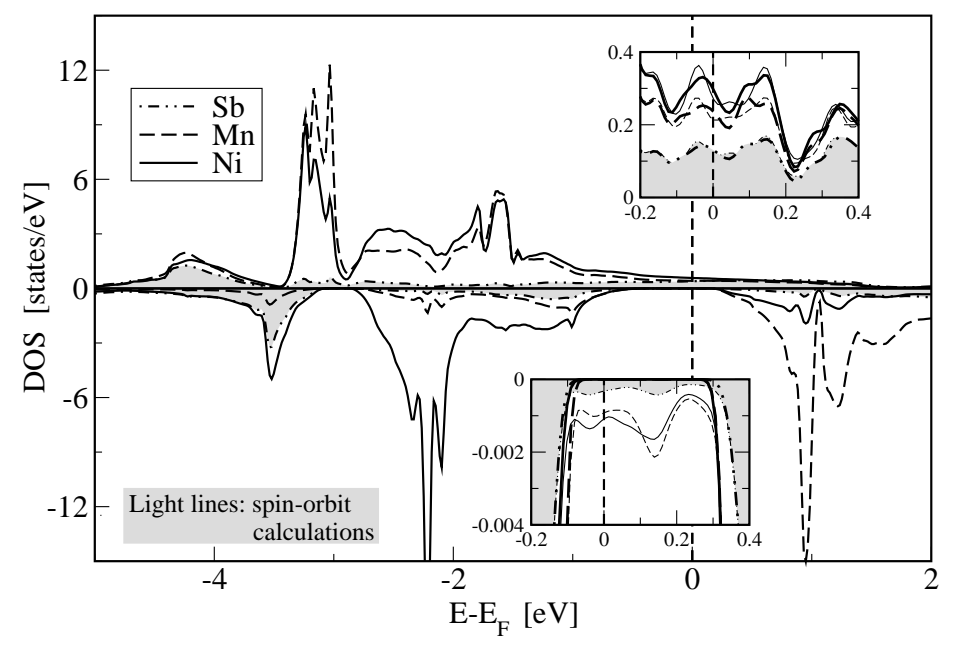

Figure 2. Atom-projected density of states (DOS) of bulk NiMnSb. The DOS in the interstitial region is not shown. The insets show magnifications of the area around the gap; with the light lines we indicate also the calculations where spin-orbit coupling has been included.

of a normal metal with the Fermi level crossing the $d$-like bands. On the contrary the minority-spin bands are like in a semiconductor and the Fermi level lies in a gap. Notice that the gap is an indirect one between the $\Gamma$ and $X$ points in the reciprocal b.c.c. unit cell.

The character of each band has been extensively discussed in reference [10. The lower bands in the figure $\square$ arise from the $p$-states of $\mathrm{Sb}$ and the minority-spin gap is created between the bonding and antibonding $d$-hybrids created by the $\mathrm{Mn}$ and $\mathrm{Ni}$ atoms. The minority-spin bonding states have most of their weight at the $\mathrm{Ni}$ atom and the antibonding at the Mn atom leading to very large localised spin moments at the $\mathrm{Mn}$ atoms 36. There are exactly 9 occupied minority states and the total spin moment follows the Slater-Pauling behaviour shown in reference [10, being exactly $4 \mu_{B}$.

In figure 2 we present the atom-projected density of states (DOS) which is defined by the muffin-tin spheres surrounding each atom. We do not show the DOS for the interstitial region (i.e. between the muffin-tin spheres) and the $\mathrm{Sb} s$-states low in energy. As we just discussed the lowest part of the DOS are occupied by the $p$ states of $\mathrm{Sb}$ which couple also to $p$-states at the other sites. Ni and $\mathrm{Mn}$ atoms create a common majority $d$ band while, as we mentioned, the minority occupied $d$-like hybrids are mainly located at the $\mathrm{Ni}$ atom leading to a large Mn spin moment $\left(3.729 \mu_{B}\right)$ and a small Ni one $\left(0.246 \mu_{B}\right)$. The Sb moment is very small $\left(-0.066 \mu_{B}\right)$ and antiparallel to the spin moments of both $\mathrm{Ni}$ and $\mathrm{Mn}$. Atomic spin moments are obtained by integrating over the non-overlapping muffin-tin spheres, so that the sum of the atomic moments is not exactly $4 \mu_{B}$.

All calculations are done within the scalar-relativistic approximation. Inclusion of spin-orbit coupling will couple the two spin-directions and partially fill the minority 

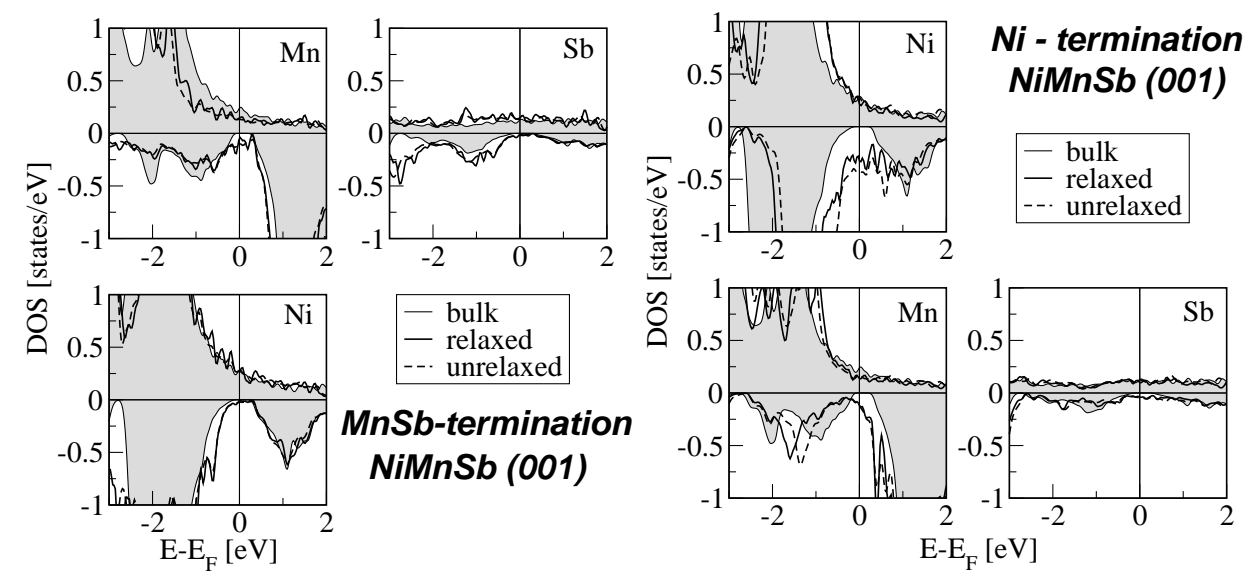

Figure 3. Local DOS for the atoms at the surface and subsurface layers for both $\mathrm{Ni}$ - and $\mathrm{MnSb}$ - terminated $\mathrm{NiMnSb}(001)$ surfaces. The results of the relaxed and unrelaxed surfaces are indicated by thick solid lines and dashed lines, respectively. Grey shaded regions represent the bulk results.

gap. Performing calculations that include spin-orbit coupling self-consistently, we find that the overall DOS and spin-moments scarcely changed and thus we present the results only around the gap in the insets of figure 2 The majority-spin DOS around the Fermi level changes only marginally while states are now present within the gap. But the intensity of the minority-spin DOS is two orders of magnitude smaller than the majority-spin DOS at the Fermi level and instead of a gap there is now a region of almost $100 \%$ spin-polarization. These results agree with the work presented in reference [37. The DOS induced by minority-spin surface states will be much more important than the contribution of spin-orbit coupling, thus the latter quantity can be neglected when studying the surface properties of NiMnSb. The orbital magnetism in NiMnSb is discussed in reference [38.

\section{4. (001) surfaces}

\subsection{Structure and relaxation}

As described in section 2] there are two different terminations for the (001) surfaces: a $\mathrm{Ni}$ or a MnSb layer. In both cases the three top layers were relaxed. In the case of the Ni termination, almost no buckling or relaxation of the MnSb subsurface layer was observed, while the distance between the top Ni layer and the subsurface layer was reduced by around $10 \%$. In the case of the $\mathrm{MnSb}$ termination, the $\mathrm{Mn}$ atom at the surface layer moves inwards and the $\mathrm{Sb}$ atom outwards. The distance between the $\mathrm{Mn}$ surface atom and the Ni subsurface layer is contracted by $3.5 \%$ and the distance between the Sb surface atom and the Ni subsurface layer is expanded by $7.3 \%$. Qualitatively, these results agree with the results obtained for the $\mathrm{MnSb}$ termination of the (001) surface by Jenkins and King [29]. 
$(00$;

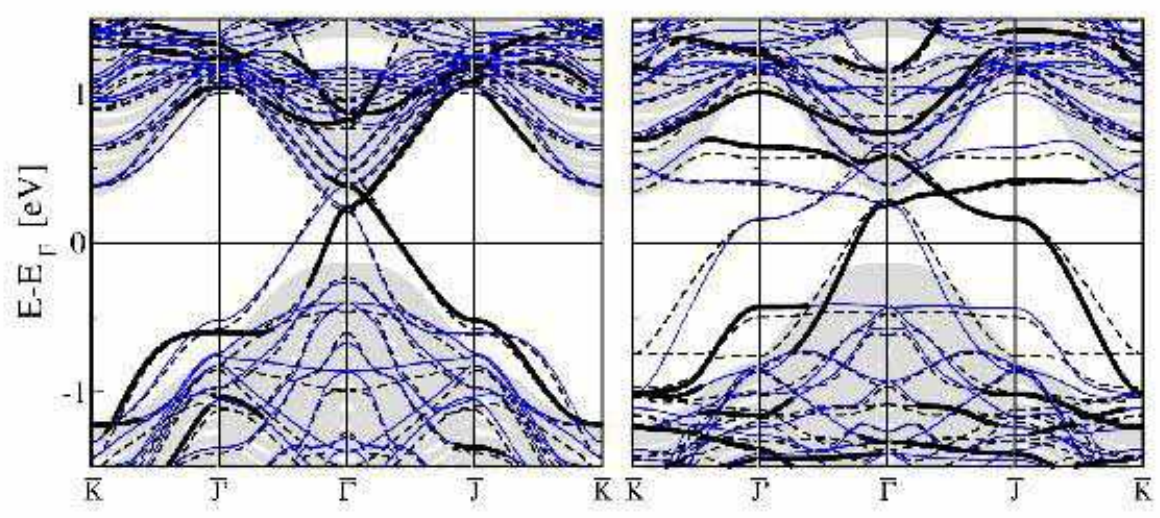

Figure 4. Minority-spin surface bandstructure for the MnSb (left) and $\mathrm{Ni}$ (right) terminated (001) surfaces. Grey regions indicate the projected bulk bandstructure. Dashed lines show the bandstructure for the unrelaxed surface, while thin full lines indicate the result for the relaxed surface. Thick lines indicate surface states on one of the of the two equivalent surfaces of the film (we considered that a surface state should have more than $50 \%$ of its weight located at the first two surface layers).

\subsection{Density of states and bandstructures}

In the right panel of figure 3 we present the atom- and spin-projected densities of states for the Ni atom at the surface and the Mn and Sb atoms in the subsurface layer for the case of the Ni-terminated surface. The left panel contains the results for the $\mathrm{MnSb}$ terminated $\mathrm{NiMnSb}(001)$ surface. For both possible terminations we include the surface DOS of both the relaxed and unrelaxed calculations together with the bulk results (grey region). We see that relaxation has a very small effect on the DOS even around the Fermi level. In the following, only the results including relaxation will be discussed.

In the case of the MnSb terminated surface, the DOS with the exception of the gap area is very similar to the bulk calculations. The Ni atom in the subsurface layer presents practically half-metallic character with an almost zero minority-spin DOS, while for the bulk there is an absolute gap. The $\mathrm{Mn}$ and $\mathrm{Sb}$ atoms in the surface layer show more pronounced differences with respect to the bulk, and within the gap there is a very small Mn- $d$ and Sb- $p$ DOS. These intensities are due to the two surface states discussed already by Jenkins and King [29. These states are strongly localised at the surface layer as in the subsurface layer there are practically no states inside the gap. This is in agreement with previous first principles calculations by Galanakis [25]. Our theoretical results agree with the experiments of Ristoiu et al. [24] who in the case of a MnSb well ordered (001) surface measured a high spin-polarization.

To examine the origin of these surface states we also calculated the surface bandstructures shown in figure 4. Here, the two-dimensional Brillouin zone is a square. Relaxations (thin solid lines) give rise to only small changes with respect to the unrelaxed results (dashed lines). Since the films in our calculations have two surfaces that are rotated by $90^{\circ}$ with respect to each other, the surface bandstructure seems to have a four-fold symmetry axis through the $\bar{\Gamma}$ point. Thick solid lines denote surface states arising from only one surface. Our results agree with the ones of Jenkins 
Table 1. Spin-projected DOS at the Fermi level $\left(n^{\uparrow}\left(E_{\mathrm{F}}\right)\right.$ or $\left.n \downarrow\left(E_{\mathrm{F}}\right)\right)$ for different (001) surfaces taking into account either the top two layers, $S$ and $S-1$ (upper panel), or the top four layers (lower panel). The spin-polarization is defined as $P=\frac{n^{\uparrow}\left(E_{\mathrm{F}}\right)-n^{\downarrow}\left(E_{\mathrm{F}}\right)}{n^{\uparrow}\left(E_{\mathrm{F}}\right)+n \downarrow\left(E_{\mathrm{F}}\right)}$

\begin{tabular}{ll|rr|rr}
\hline $\begin{array}{l}\text { Spin } \\
\text { Polarisation }\end{array}$ & $\begin{array}{r}(001) \mathrm{Ni} \\
\text { unrelaxed }\end{array}$ & $\begin{array}{r}(001) \mathrm{Ni} \\
\text { relaxed }\end{array}$ & $\begin{array}{r}(001) \mathrm{MnSb} \\
\text { unrelaxed }\end{array}$ & $\begin{array}{r}(001) \mathrm{MnSb} \\
\text { relaxed }\end{array}$ \\
\hline Layers & $n^{\uparrow}\left(E_{\mathrm{F}}\right)$ & 0.855 & 0.641 & 0.777 & 0.796 \\
$\mathrm{~S}, \mathrm{~S}-1$ & $n^{\downarrow}\left(E_{\mathrm{F}}\right)$ & 0.655 & 0.556 & 0.161 & 0.107 \\
& $P_{1}$ & $13 \%$ & $7 \%$ & $66 \%$ & $76 \%$ \\
\hline Layers & $n^{\uparrow}\left(E_{\mathrm{F}}\right)$ & 1.781 & 1.352 & 1.573 & 1.543 \\
$\mathrm{~S}, \mathrm{~S}-1$ & $n^{\downarrow}\left(E_{\mathrm{F}}\right)$ & 0.730 & 0.618 & 0.194 & 0.135 \\
$\mathrm{~S}-2, \mathrm{~S}-3$ & $P_{2}$ & $42 \%$ & $37 \%$ & $78 \%$ & $84 \%$ \\
\hline
\end{tabular}

and King who - for the same surface - have shown that there are two surface states [29. They noted that the lower lying state $\left(0.20 \mathrm{eV}\right.$ above the Fermi level, $\left.E_{\mathrm{F}}\right)$ is due to the interaction between $e_{g}$-like dangling bond states located at the Mn atoms, while the second surface state, $\left(\sim 0.3 \mathrm{eV}\right.$ above $\left.E_{\mathrm{F}}\right)$ arises from the hybridization between $t_{2 g}$-like orbitals of $\mathrm{Mn}$ with $p$-type orbitals of $\mathrm{Sb}$. The first surface state disperses downwards along the $\overline{\Gamma J}$ direction while the second surface state disperses upwards along the same direction. Since their dispersion reverses along the $\overline{\Gamma J^{\prime}}$ direction, we assume that there is also significant interaction with the subsurface $(\mathrm{Ni})$ layer. The two surface states cross along the $\overline{\Gamma J}$ direction bridging the minority gap between the valence and the conduction band. Along the other directions anticrossing occurs leading to band-gaps. Of interest are also the saddle-like structures around the zone center which show up as van Hove singularities in the DOS.

The $\mathrm{Ni}$ terminated surface also shows two surface states, as can be seen from figure 4 In comparison to the MnSb termination, this more open surface leads to rather flat dispersions of the surface states. Accordingly in the DOS, shown in figure 3 , these surface states are much more intense and effectively destroy the minority gap on this surface.

\subsection{Spin-polarization and magnetic moments}

Using the above results, the spin-polarization at the Fermi level can be determined. At interfaces, it is of prime importance since in a current-injection experiment normally the electrons near the Fermi level are involved [3]. In figure 5 we have gathered the angular-momentum spin- and layer-projected DOS at the Fermi level $\left(n^{\uparrow}\left(E_{\mathrm{F}}\right)\right.$ or $\left.n^{\downarrow}\left(E_{\mathrm{F}}\right)\right)$ for all the calculated films. The layers near the edges of each figure represent the two equivalent surfaces while the layers at the middle are bulk-like. In the case of the unrelaxed surfaces (upper panels) the minority DOS of the layers at the middle of the film is almost zero and thus the thickness of the film used in the calculation is sufficient to realistically represent the real surface. Only in the case of the relaxated MnSb-terminated surface, the Mn atom at the middle layer presents a very small DOS. As expected, the states at the Fermi level are mainly of $d$ character for $\mathrm{Mn}$ and $\mathrm{Ni}$ and of $p$-type for $\mathrm{Sb}$ for both spin-directions.

To expand our conclusions we need to quantify the DOS at the Fermi level and in table 1 we have gathered the results for all surfaces. We have calculated the spin- 

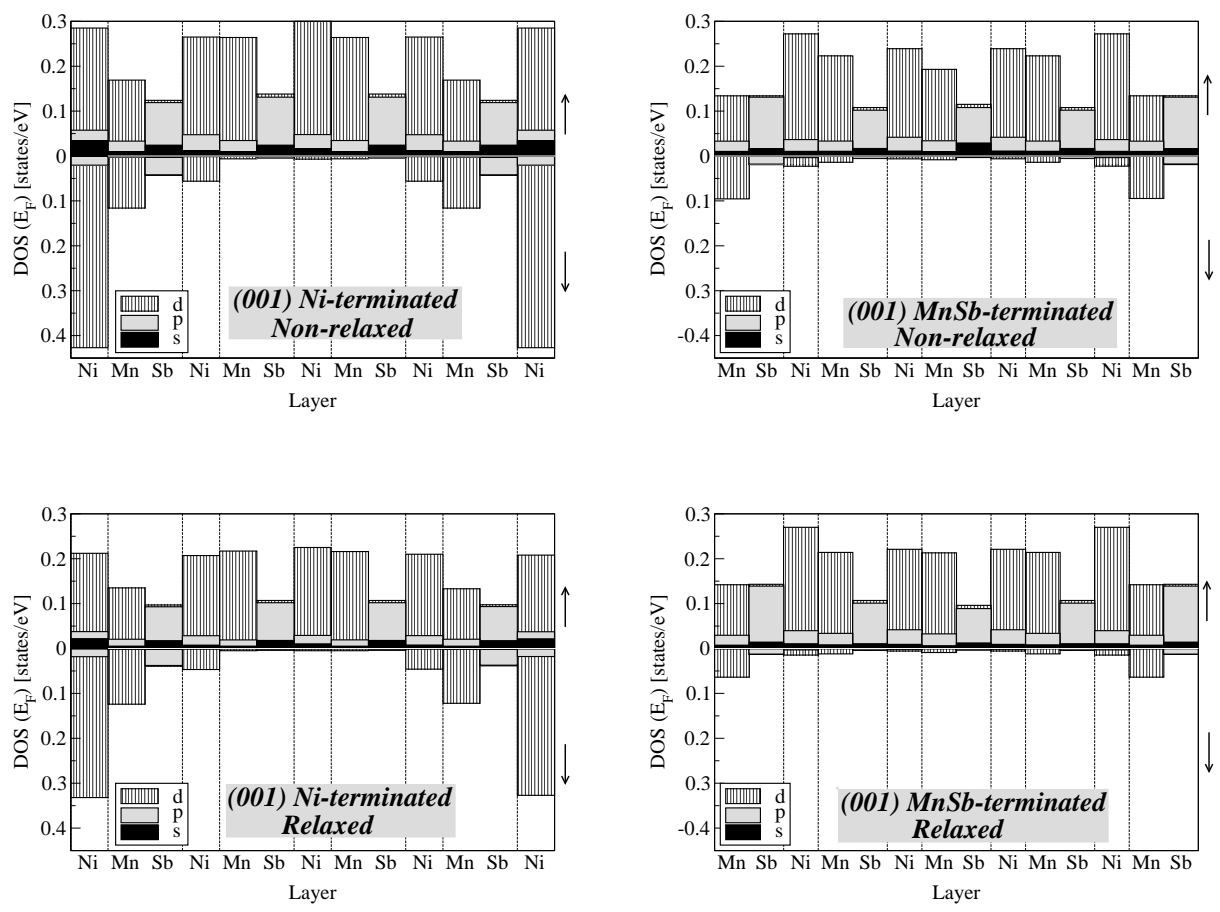

Figure 5. Atom- and angular momentum-projected DOS at the Fermi level for the different layers of the unrelaxed (top) and relaxed (bottom) film with $\mathrm{Ni}$ (left) and $\mathrm{MnSb}$ (right) termination. Note, that the films in the case of the (001) surfaces are inversion-symmetric.

polarization either taking into account only the first two surface layers $P_{1}$ or the first four surface layers $P_{2} . \quad P_{2}$ represents quite well the experimental situation as the spin-polarization in the case of films is usually measured by inverse photoemission which probes the first few surface layers of the sample 39. As expected, the inclusion of more layers increases the spin-polarization since deeper layers are more bulk-like. Relaxation in the case of the Ni-terminated surface decreases the spin-polarization while in the case of the MnSb-terminated surface the spin-polarization is increased by the relaxation of the atomic positions.

In the case of the Ni terminated surface, the minority-spin DOS at the Fermi level is quite large with respect to the majority DOS and net polarization $P_{2}$ is $42 \%$ for the unrelaxed case and slightly decreases to $37 \%$ by structural optimization. In the case of the $\mathrm{MnSb}$ terminated surface the spin-polarization is much larger and now $P_{2}$ reaches a value of $84 \%$ for the relaxed structure, which means that more than $90 \%$ of electrons at the Fermi level are of majority-spin character. Our values for $P_{1}$ can be compared to reference [25], where $\sim 0 \%$ and $38 \%$ spin-polarisation have been found for the $\mathrm{Ni}$ and $\mathrm{MnSb}$ terminations, respectively. As can be seen from figure[5 the main difference between the two different terminations is the contribution of the $\mathrm{Ni}$ spin-down states. In the case of the MnSb surface the $\mathrm{Ni}$ in the subsurface layer has a negligible DOS at the Fermi level with respect to the Ni-terminated surface. It is interesting also to see 
Table 2. Atom-projected spin magnetic moments $\left(m_{\text {spin }}\right)$ in $\mu_{B}$ for the atoms at the top four layers for both Ni- and MnSb-terminated (001) surfaces for both relaxed and unrelaxed cases.

\begin{tabular}{|c|c|c|c|c|c|c|c|}
\hline \multicolumn{3}{|c|}{$\begin{array}{c}\text { Ni-terminated } \\
\text { unrel. }\end{array}$} & rel. & \multicolumn{3}{|c|}{$\begin{array}{c}\text { MnSb-terminated } \\
\text { unrel. }\end{array}$} & rel. \\
\hline $\mathrm{Ni}$ & (S) & 0.44 & 0.38 & $\mathrm{Mn}$ & $(\mathrm{S})$ & 3.94 & 3.93 \\
\hline $\mathrm{Mn}$ & $(\mathrm{S}-1)$ & 3.79 & 3.64 & $\mathrm{Sb}$ & (S) & -0.10 & -0.10 \\
\hline $\mathrm{Sb}$ & $(\mathrm{S}-1)$ & -0.04 & -0.04 & $\mathrm{Ni}$ & $(S-1)$ & 0.21 & 0.24 \\
\hline $\mathrm{Ni}$ & $(\mathrm{S}-2)$ & 0.27 & 0.28 & $\mathrm{Mn}$ & $(\mathrm{S}-2)$ & 3.66 & 3.69 \\
\hline $\mathrm{Mn}$ & $(\mathrm{S}-3)$ & 3.71 & 3.59 & $\mathrm{Sb}$ & $(\mathrm{S}-2)$ & -0.07 & -0.07 \\
\hline $\mathrm{Sb}$ & $(\mathrm{S}-3)$ & -0.06 & -0.05 & $\mathrm{Ni}$ & $(\mathrm{S}-3)$ & 0.27 & 0.24 \\
\hline
\end{tabular}

from this figure, that for both terminations the spin-polarization of the Mn near the surface at the Fermi level is close to zero while $\mathrm{Sb}$ atoms in both cases show a large spinpolarization. The calculated $P_{2}$ value of $84 \%$ for the $\mathrm{MnSb}$ terminated surface is larger than the experimental value of $67 \%$ obtained by Ristoiu and collaborators [24] for a thin-film terminated in a MnSb stoichiometric alloy surface layer. A direct comparison between experiment and theory is not straightforward, since experimentally different layers will contribute with different weight to the spin-polarization.

In table 2 we have gathered the spin magnetic moments of the atoms in the surface and subsurface layers. We should notice that relaxation has in most cases only a small effect on the spin moments. Even for the surface layer which shows the largest relaxation effects, spin moments change by at most $0.06 \mu_{B}$.

In the case of the MnSb terminated $\mathrm{NiMnSb}(001)$ surface, the surface layer loses $\sim 0.3 e^{-}$as compared to the bulk. This is due to the spilling out of charge into the vacuum and affects mainly the Mn minority-spin electrons. Therefore, the Mn's spin magnetic moment increases with respect to the bulk and is slightly more than $3.9 \mu_{B}$. This behaviour arises from the reduced symmetry of the Mn atom in the surface which loses two of the four neighbouring Ni atoms. In the majority band this leads to a narrowing of the $d$-DOS and this affects also the subsurface Ni layer that loses $0.1 e^{-}$, while in the minority valence band the Mn $d$-contribution decreases by $0.2 e^{-}$. Moreover, the splitting between the unoccupied Mn states above $E_{\mathrm{F}}$ and the center of the occupied Mn states decreases and at $E_{\mathrm{F}}$ a surface states appears. We should also mention here that in the case of a half-metallic material the total spin magnetic moment per unit cell should be an integer since the number of both the total valence electrons and the minority-spin occupied states are integers; the spin moment in $\mu_{B}$ is simply the number of uncompensated spins, i.e. $4 \mu_{B}$. In the case of the surfaces the half-metallic character is lost and an increase of the total spin moment is observed, which is no more an integer number.

In the case of the Ni terminated surface, the changes in the DOS compared to the bulk are more pronounced. The Ni atom in the surface loses some charge. As was the case for the Mn surface atom in the MnSb terminated surface, also the surface atoms spin magnetic moment is increased (see table 2). The $\mathrm{Mn}$ and $\mathrm{Sb}$ atoms in the subsurface layer present a charge transfer comparable to the bulk compound and also a comparable spin moment. 
Table 3. Relative changes in the distance $\Delta d_{i j}$ between successive layers $i, j$ when the atomic positions were relaxed for the (111) surfaces. Negative signs correspond to contractions, positive to expansions.

\begin{tabular}{l|rrr}
\hline & $\Delta d_{12}$ & $\Delta d_{23}$ & $\Delta d_{34}$ \\
\hline Ni-Sb-Mn-... & $-23 \%$ & $2 \%$ & $<1 \%$ \\
Ni-Mn-Sb-... & $-18 \%$ & $4 \%$ & $-3 \%$ \\
Mn-Ni-Sb-... & $-13 \%$ & $-5 \%$ & $2 \%$ \\
Mn-Sb-Ni-... & $-16 \%$ & $18 \%$ & $\sim 0 \%$ \\
Sb-Ni-Mn-... & $2 \%$ & $-11 \%$ & $4 \%$ \\
Sb-Mn-Ni-... & $-16 \%$ & $32 \%$ & $-7 \%$ \\
\hline
\end{tabular}

\section{5. (111) surfaces}

\subsection{Structure and relaxation}

In this section, we will study the (111) surfaces of NiMnSb. Relaxations in the case of the (111) surfaces are considerably larger than for the (001) ones. In table 3 we have gathered the change in the distance between two successive layers with respect to the unrelaxed cases. The closed packed (111) layers contain only one chemical element. Note, that in the unrelaxed cases the distance between $\mathrm{Sb}$ and $\mathrm{Mn}$ successive layers is twice the distance between a $\mathrm{Ni}$ and a $\mathrm{Mn}$ or $\mathrm{Sb}$ layer.

When the (111) surface is Ni-terminated, the $\mathrm{Ni}$ atoms at the surface layer move closer to the subsurface layer and the contraction is $23 \%$ and $18 \%$ for $\mathrm{Sb}$ and $\mathrm{Mn}$ as subsurface layers, respectively. Relaxations are much less important deeper than the surface layer. When the surface is Mn terminated with a Mn-Ni-Sb-... stacking sequence, the $\mathrm{Mn}$ atoms move closer to $\mathrm{Ni}$ due to the lower coordination. In the case of a Mn-Sb-Ni-... sequence, relaxations are more important since the Mn-Sb distance is twice the Mn-Ni one. From table 3 we see, that this results not only in a large contraction of the first two layers (negative $\Delta d_{12}$ ), but also in a expansion of the next interlayer distance, $\Delta d_{23}$. A similar effect can be observed on the Sb-Mn-Ni-... terminated surface. In the latter case, similar relaxations have also been obtained by Jenkins 30.

\subsection{Density of states and bandstructures}

In figure [6] we have gathered the spin-resolved density of states (DOS) for the three layers closest to the surface for both types of Ni termination. For the Ni-Mn-Sb... termination, there is a minority surface state pinned exactly at the Fermi level which completely destroys the half-metallicity. The population of the majority states increases and due to the exchange splitting the minority states are pushed higher in energy. This results in a very sharp shape of the surface state. Actually there are two surface states as we will discuss later in this paragraph. This phenomenon is more pronounced for the Mn atom at the subsurface layer, whose occupied minority states have a small weight, and thus it presents a much larger exchange splitting energy since this one scales with the spin magnetic moment. This surface state gradually decays and for the $\mathrm{Ni}$ atom at the $\mathrm{S}-3$ position (not shown here) it practically vanishes. We can identify this surface states also in the surface band-structure presented in the upper left of figure 7 Thick lines mark the surface states for this termination. We 


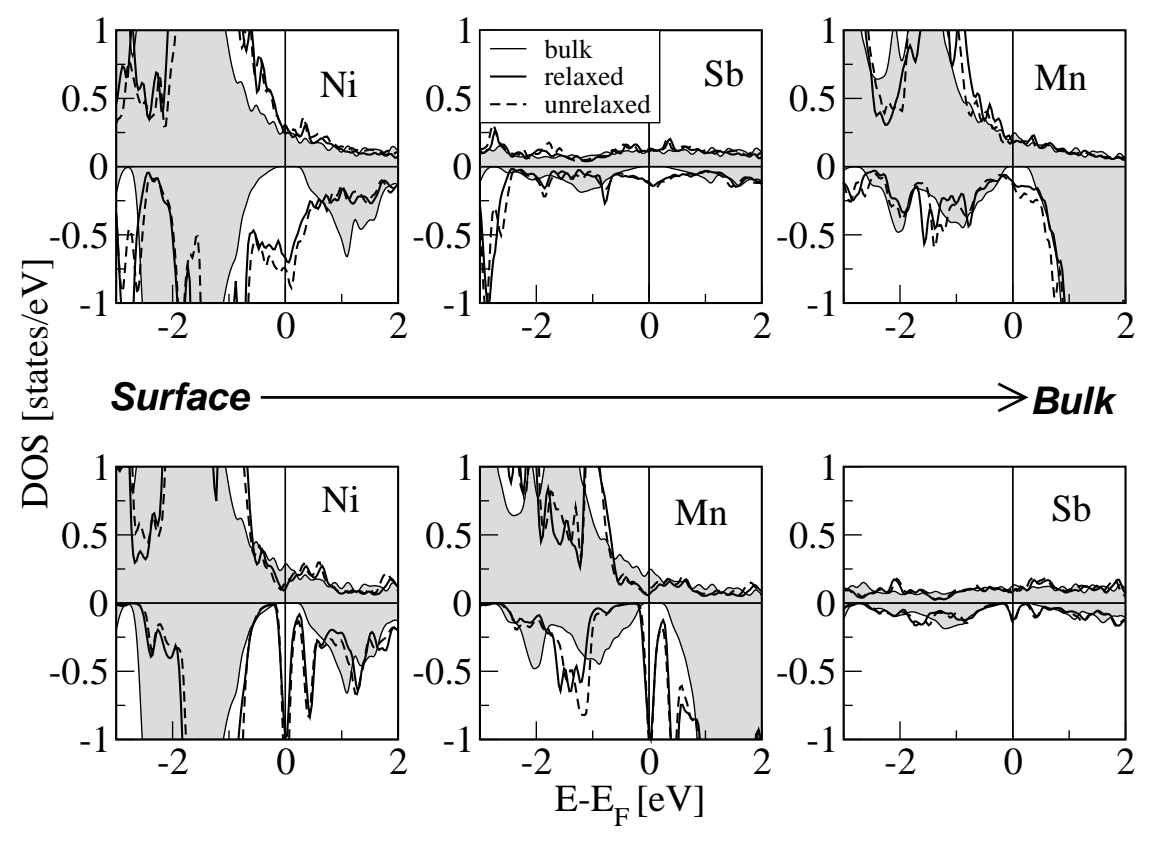

Figure 6. Same as figure 3 for the Ni-terminated (111) surfaces. There are two different $\mathrm{Ni}$ terminations, either with a $\mathrm{Sb}$ or a $\mathrm{Mn}$ layer as the subsurface one.

observe now two surface states, similar to the (001) surfaces, which are very narrowspread in energy around the Fermi level, resulting in a very sharp peak structure.

In the case of the Ni-Sb-Mn-... surface, the $\mathrm{Ni}$ bands even move slightly higher in energy. Therefore, the Ni spin moment is much smaller and the Mn atom is deep in the substrate. The surface states are now much more extended in the energy axis and cannot be well separated from the rest of the DOS as shown in figure 6 This situation is similar to the Ni terminated (001) surface. These surfaces states (figure 7) are clearly much broader in energy than the states in the case of the Ni-Mn-Sb-... termination resulting in a very extended peak at the Fermi level which is not easily distinguished in the DOS. Our band-structure is similar to the one calculated by Jenkins 30].

In figure 8 we have gathered the DOS for the first three layers for all Mn and $\mathrm{Sb}$ surfaces. In the case of the Mn-terminated surfaces, there is a minority surface state pinned exactly at the Fermi level which destroys the half-metallicity and which is also visible in the $\mathrm{Ni}$ subsurface layer, but vanishes in the next Mn layer (not shown here). The overall DOS are similar to the bulk case and the increase of the Mn spin moment at the interface reflects that high-lying majority antibonding $d$-states, which are above the Fermi level in the bulk but now move below it, push also the majority bands somewhat lower in energy [10]. The surface states in the reciprocal space are similar to the Ni-Mn-Sb-... case shown in figure 7 In the case of the Mn-Sb-Ni-... surface, there are three surface states with very flat dispersion while in the case of the Mn-Ni-Sb-... (111) surface there is just one very flat surface state centered along the 


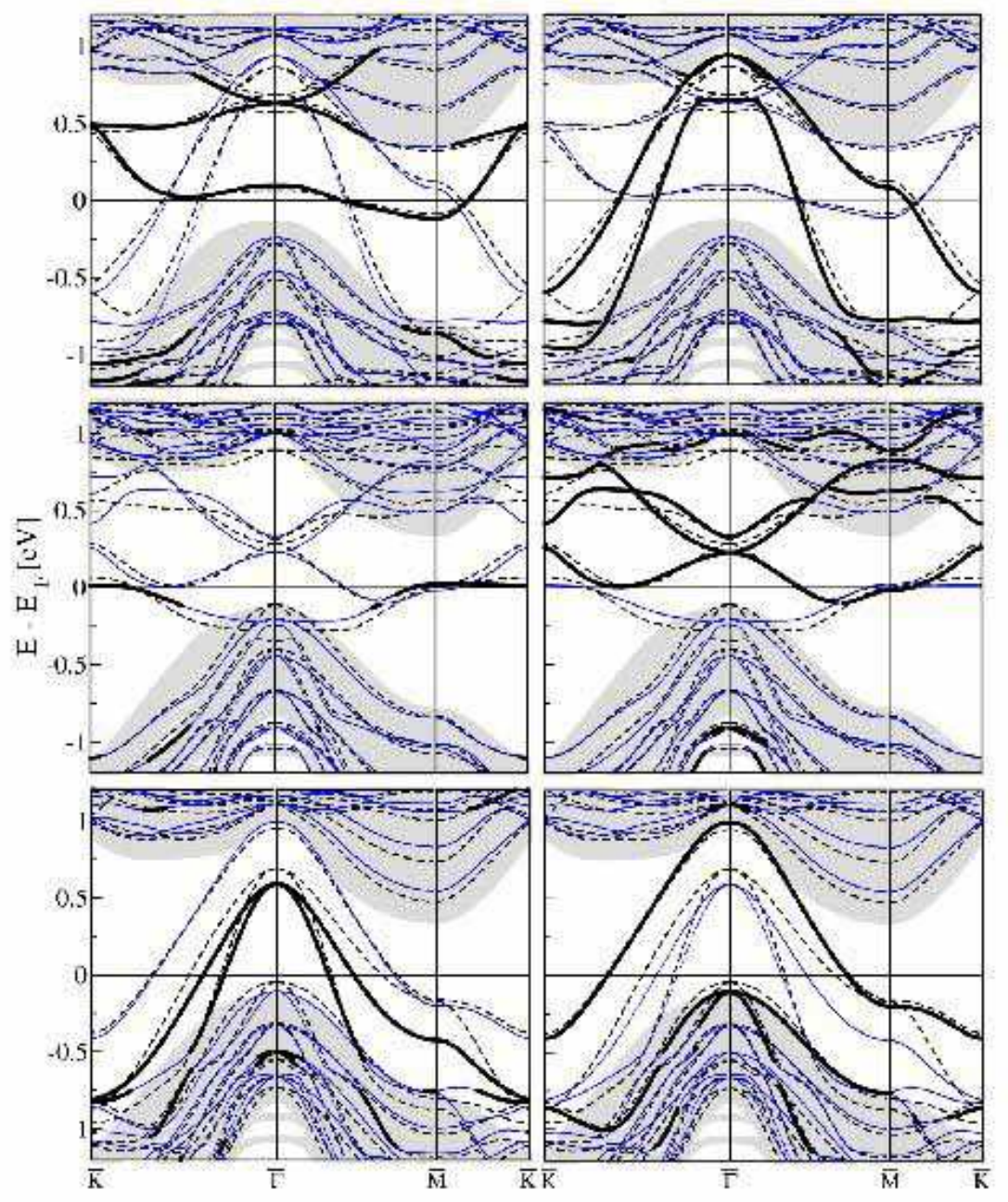

Figure 7. Surface bandstructures for the Ni (top) Mn (middle) and Sb (bottom) terminated (111) oriented NiMnSb films. The two surfaces of the film have different subsurface layers and, therefore, give rise to different surface states. The left column shows the surface states of the Ni-Mn-Sb-..., Mn-Ni-Sb-... and Sb$\mathrm{Mn}-\mathrm{Ni}-\ldots$ terminated surfaces (top to bottom), while on the right the states from the Ni-Sb-Mn-..., Mn-Sb-Ni-... and Sb-Ni-Mn-... surfaces are marked. Otherwise the labeling is identical to figure 4

$\overline{M K}$ line leading to the very sharp peak shown in figure 8 and leaving a band gap just above the Fermi level. Also in the case of the Sb terminated surface there is a minority surface state slightly below the Fermi level which also destroys the half-metallicity at the surface. Its intensity is large also for the $\mathrm{Ni}$ at the subsurface layer but already for the Mn atom it starts to smear out. These surface states can also be traced in the reciprocal space as shown in the lower panels of figure 7 They are very wide in energy and thus can not be well separated from the rest of the DOS. Where comparable, our surface band-structures agree with the results of Jenkins 30. 

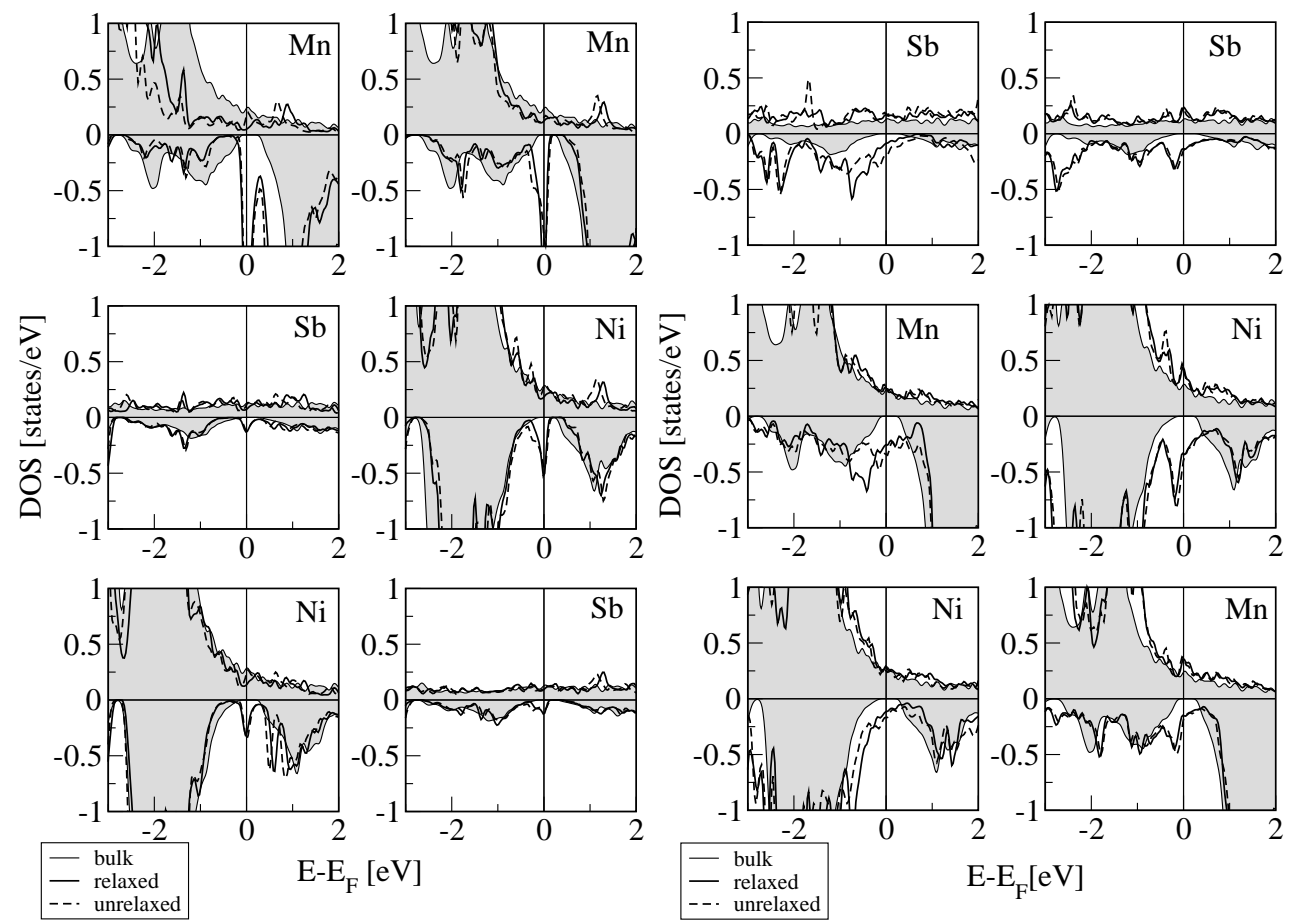

Figure 8. Same as figure 6 for the Mn- and Sb-terminated (111) surfaces. Each column represents a different surface termination. The top panels represent the surface layers, the middle ones the subsurface layers and the bottom panels the subsubsurface ones.

\subsection{Spin-polarization and magnetic moments}

In the bulk case $\mathrm{Ni}$ has four $\mathrm{Mn}$ and four $\mathrm{Sb}$ atoms as first neighbours. At the $\mathrm{Ni}$ terminated (111) surface, the $\mathrm{Ni}$ atom at the surface loses four out of its eight first neighbours. In the case of the Ni-Mn-Sb-... termination it loses three $\mathrm{Sb}$ atoms and one $\mathrm{Mn}$ atom while in the Ni-Sb-Mn-... case one $\mathrm{Sb}$ and three $\mathrm{Mn}$ atoms.

In table 4 we have gathered the spin moments for the first six layers for all surfaces under study. Generally, we can observe that relaxations tend to decrease the Mn moments, while in some cases the $\mathrm{Ni}$ or $\mathrm{Sb}$ moments can increase slightly. In the case of the Ni-Mn-Sb-... termination, both $\mathrm{Ni}$ and $\mathrm{Mn}$ atoms at the surface have very large moments with respect to both the bulk calculations and the Ni-Sb-Mn-... case. Especially the $\mathrm{Ni}$ moment is almost doubled $\left(0.47 \mu_{B}\right)$ with respect to the bulk value of $0.25 \mu_{B}$. In the bulk NiMnSb the minority gap is created by the hybridization between the $d$-orbitals of the $\mathrm{Ni}$ and $\mathrm{Mn}$ atoms, but the Sb atom plays also a crucial role since it provides states lower in energy than the $d$ bands which accommodate electrons of the transition metal atoms [10. At the Ni-Mn-Sb-... terminated surface, each $\mathrm{Ni}$ surface atoms loses three out of the four $\mathrm{Sb}$ first neighbours and they regain most of the charge accommodated in the $p$-bands of Sb. These extra electrons fill up mostly majority states, increasing the $\mathrm{Ni}$ spin moment. Mn spin moment is also increased since $\mathrm{Mn}$ and $\mathrm{Ni}$ majority $d$-states strongly hybridize forming a common majority band as it was shown in reference [10. Thus the spin moment of Mn at the 
Table 4. Atom-projected spin magnetic moments $\left(m_{\text {spin }}\right)$ in $\mu_{B}$ for the atoms at the top six layers for all $\mathrm{Ni}$-, Mn- and Sb-terminated (111) surfaces and for both relaxed and unrelaxed cases.

\begin{tabular}{|c|c|c|c|c|c|c|c|c|}
\hline & $\begin{array}{l}\text { Ni-Mn-Sb-... } \\
\text { unrel. }\end{array}$ & rel. & & $\begin{array}{l}\text { n-Ni-Sb-... } \\
\text { unrel. }\end{array}$ & rel. & & $\begin{array}{l}\text { Sb-Mn-Ni-... } \\
\text { unrel. }\end{array}$ & rel. \\
\hline $\mathrm{Ni}(\mathrm{S})$ & 0.54 & 0.47 & $\operatorname{Mn}(\mathrm{S})$ & 3.90 & 3.73 & $\mathrm{Sb}(\mathrm{S})$ & -0.19 & -0.21 \\
\hline $\mathrm{Mn}$ & 3.89 & 3.77 & $\mathrm{Ni}$ & 0.23 & 0.28 & $\mathrm{Mn}$ & 3.62 & 3.56 \\
\hline $\mathrm{Sb}$ & -0.05 & -0.05 & $\mathrm{Sb}$ & -0.07 & -0.07 & $\mathrm{Ni}$ & 0.19 & 0.09 \\
\hline $\mathrm{Ni}$ & 0.27 & 0.27 & Mn & 3.63 & 3.56 & $\mathrm{Sb}$ & -0.07 & -0.07 \\
\hline $\mathrm{Mn}$ & 3.70 & 3.57 & $\mathrm{Ni}$ & 0.24 & 0.25 & Mn & 3.65 & 3.59 \\
\hline \multirow[t]{2}{*}{$\mathrm{Sb}$} & -0.05 & -0.05 & $\mathrm{Sb}$ & -0.07 & -0.08 & $\mathrm{Ni}$ & 0.26 & 0.24 \\
\hline & $\begin{array}{l}\text { Ni-Sb-Mn-... } \\
\text { unrel. }\end{array}$ & rel. & & $\begin{array}{l}\text { n-Sb-Ni-... } \\
\text { unrel. }\end{array}$ & rel. & & $\begin{array}{l}\text { Sb-Ni-Mn-... } \\
\text { unrel. }\end{array}$ & rel. \\
\hline $\mathrm{Ni}(\mathrm{S})$ & 0.30 & 0.30 & $\operatorname{Mn}(\mathrm{S})$ & 4.16 & 3.89 & $\mathrm{Sb}(\mathrm{S})$ & -0.12 & -0.12 \\
\hline $\mathrm{Sb}$ & -0.04 & -0.04 & $\mathrm{Sb}$ & -0.04 & -0.05 & $\mathrm{Ni}$ & 0.13 & 0.15 \\
\hline $\mathrm{Mn}$ & 3.71 & 3.54 & $\mathrm{Ni}$ & 0.30 & 0.33 & $\mathrm{Mn}$ & 3.52 & 3.50 \\
\hline $\mathrm{Ni}$ & 0.23 & 0.21 & $\mathrm{Mn}$ & 3.70 & 3.66 & $\mathrm{Sb}$ & -0.06 & -0.07 \\
\hline $\mathrm{Sb}$ & -0.07 & -0.07 & $\mathrm{Sb}$ & -0.06 & -0.07 & $\mathrm{Ni}$ & 0.26 & 0.24 \\
\hline Mn & 3.68 & 3.56 & $\mathrm{Ni}$ & 0.27 & 0.27 & $\mathrm{Mn}$ & 3.70 & 3.69 \\
\hline
\end{tabular}

subsurface layer increases to $3.77 \mu_{B}\left(3.89 \mu_{B}\right.$ in the unrelaxed case) with respect to the bulk value of $3.73 \mu_{B}$. If one goes further away from the surface, the atoms have a bulklike environment and their spin moments are similar to the bulk moments. In the Ni-Sb-Mn-... surface, $\mathrm{Ni}$ at the surface loses only one Sb first neighbour and the effect of the cut-off neighbours is much smaller. The moment is slightly smaller than the bulk one mainly due to a surface state at the minority band shown in figure 6 . Already the $\mathrm{Sb}$ subsurface atom regains a bulklike behaviour for the spin moment.

In the case of the Mn surfaces, $\mathrm{Mn}$ at the surface layer loses half of its $\mathrm{Sb}$ second neighbours. Similarly to what happened in the case of the Ni-Mn-Sb-... surface, its spin moment is strongly enhanced especially in the Mn-Sb-Ni-... case (to $3.89 \mu_{B}$ ). In this case, we can think that Mn has a subsurface layer made up by voids and thus the hybridization between the $\mathrm{Mn} d$-orbitals and the $\mathrm{Sb} p$ - and $\mathrm{Ni} d$-orbitals is strongly reduced leading to an increase of its spin moment with respect to the $\mathrm{Mn}-\mathrm{Ni}-\mathrm{Sb}-. .$. case. Relaxations tend to decrease the $\mathrm{Mn}$ moment, but due to the large increase of $\Delta d_{23}$ in the $\mathrm{Mn}-\mathrm{Sb}-\mathrm{Ni}$-... case, the Ni moment increases here. The atoms deeper in the surface quickly reach a bulklike behaviour.

Following the same arguments as for $\mathrm{Mn}$, one can understand also the behaviour of the spin moments for the $\mathrm{Sb}$ terminated surfaces presented in table 4 The absolute value of the $\mathrm{Sb}$ spin moment at the surface layer increases with respect to the bulk. When the subsurface layer is a "void layer" (Sb-Mn-Ni-.. case), the hybridization effects are less important and the Sb spin moment can reach a value of $-0.2 \mu_{B}$. This is almost three times the bulk value of $-0.07 \mu_{B}$ and double the value for the (001) surface of $-0.1 \mu_{B}$. The change in the $\mathrm{Sb} p$-bands influences also through hybridization the bands of the transition metal atoms for which now the minority bands population increases leading to smaller spin moments of the $\mathrm{Ni}$ and $\mathrm{Mn}$ atoms at the subsurface layers. The phenomenon is more intense in the case of Sb-Ni-Mn-... where the $\mathrm{Ni}$ layer is just below the $\mathrm{Sb}$ surface layer and the reduction in the spin moments of $\mathrm{Ni}$ and $\mathrm{Sb}$ is much larger than in the $\mathrm{Sb}-\mathrm{Mn}-\mathrm{Ni}-\ldots$ case.

Finally, we have collected the spin-polarization at the Fermi level in table 5 
Table 5. Spin-polarization at the Fermi level for different (111) surfaces taking into account either the top three layers $\left(P_{1}\right)$ or the top six layers $\left(P_{2}\right)$. The spin-polarization is defined as in table 1

\begin{tabular}{l|rr|rr} 
& \multicolumn{2}{|c|}{$P_{1}$} & \multicolumn{2}{|c}{$P_{2}$} \\
& unrelaxed & relaxed & unrelaxed & relaxed \\
\hline Ni-Sb-Mn-... & $-13 \%$ & $-19 \%$ & $17 \%$ & $8 \%$ \\
Ni-Mn-Sb-.. & $-70 \%$ & $-74 \%$ & $-49 \%$ & $-67 \%$ \\
Mn-Ni-Sb-.. & $-64 \%$ & $-66 \%$ & $-16 \%$ & $-49 \%$ \\
Mn-Sb-Ni-.. & $-52 \%$ & $-78 \%$ & $-31 \%$ & $-58 \%$ \\
Sb-Mn-Ni-.. & $9 \%$ & $29 \%$ & $35 \%$ & $49 \%$ \\
Sb-Ni-Mn-.. & $20 \%$ & $28 \%$ & $33 \%$ & $42 \%$ \\
\hline
\end{tabular}

Since we have seen that only the $\mathrm{Sb}$ terminated surfaces have surface states that are not localised too narrow around the Fermi level, we observe only on these surfaces substantial spin-polarizations. In these cases, relaxations have the effect to increase the spin-polarization at the Fermi level, while in most other cases large negative values are observed, which get even more negative when structural relaxations are taken into account.

\section{Summary}

We have performed ab-initio calculations based on the full-potential linearised augmented plane-wave method for the (001) and (111) surfaces of the half-metallic NiMnSb Heusler alloy. The MnSb terminated (001) surfaces present electronic and magnetic properties similar to the bulk compounds. There is however a small finite Mn- $d$ and Sb- $p$ DOS within the bulk spin-down gap and these surface states are strongly localised at the surface layer. The spin-polarization at the Fermi level for this termination reaches the $84 \%$. The (001) surfaces terminated at Ni present a quite large density of states at the Fermi level and properties considerably different from the bulk and the $\mathrm{MnSb}$ terminated surfaces. In both terminations, two distinct surface states can be seen in the surface bandstructure, which are of quite different character on the two surfaces.

In all (111) surfaces minority-spin surface states destroy the half-metallicity at the surface. They are pinned at the Fermi level for the Ni and Mn terminated surfaces but are slightly below the Fermi level for the Sb terminated ones. They are localised close to the surface region and typically vanish within few atomic layers. Surface states show a variety of dispersion relations as was shown by the surface band-structures. In the case of the Ni surface with Mn as subsurface layer, Ni-Mn-Sb-..., the loss of three out of the four $\mathrm{Sb}$ first neighbours leads to a doubling of the Ni spin moment while in the Ni-Sb-Mn-... case it is near the bulk value. For the $\mathrm{Mn}$ and $\mathrm{Sb}$ terminations the lowering of the coordination increases the surface spin moments and the enhancement is larger when the subsurface layer is not a $\mathrm{Ni}$ one. Only on the $\mathrm{Sb}$ terminated surfaces substantial spin-polarizations can be observed, specifically when relaxations are taken into account.

\section{Acknowledgments}

This work was financed in part by the BMBF under auspices of the Deutsches 
Elektronen-Synchrotron DESY under contract no. $05 \mathrm{KS} 1 \mathrm{MPC} / 4$.

\section{References}

[1] Žutić I, Fabian J and Das Sarma A 2004 Rev. Mod. Phys. 76323

[2] de Boeck J, van Roy W, Motsnyi V, Liu Z, Dessein K and Borghs G 2002 Thin Solid Films 412 3; de Boeck J, van Roy W, Das J, Motsnyi V, Liu Z, Lagae L, Boeve H, Dessein K and Borghs G 2002 Semicond. Sci. Tech. 17342

[3] Wunnicke O, Mavropoulos Ph, Zeller R, Dederichs P H and Gründler D 2002 Phys. Rev. B 65 241306; Mavropoulos Ph, Wunnicke O and Dederichs Ph 2002 Phys. Rev. B 66024416

[4] Kilian K A and Victora R H 2000 J. Appl. Phys. 87 7064; Kilian K A and Victora R H 2001 IEEE Trans. Magn. $\mathbf{3 7} 1976$

[5] Tanaka C T, Nowak J and Moodera J S 1999 J. Appl. Phys. 86 6239; Tanaka C T, Nowak J and Moodera J S 1997 J. Appl. Phys. 815515

[6] Caballero J A, Park Y D, Childress J R, Bass J, Chiang W -C, Reilly A C, Pratt Jr W P and Petroff F 1998 J. Vac. Sci. Technol. A 16 1801; Hordequin C, Nozières J P and Pierre J 1998 J. Magn. Magn. Mater. 183225

[7] Webster P J and Ziebeck K R A, in Alloys and Compounds of d-Elements with Main Group Elements. Part 2., edited by H.R.J. Wijn, Landolt-Börnstein, New Series, Group III, Vol. 19,Pt.c (Springer-Verlag, Berlin), pp. 75-184.

[8] de Groot R A, Mueller F M, van Engen P G and Buschow K H J 1983 Phys. Rev. Lett. 502024

[9] Kulatov E and Mazin I I 1990 J. Phys.: Condens. Matter 2 343; Halilov S V and Kulatov E T 1991 J. Phys.: Condens. Matter 3 6363; Youn S J and Min B I 1995 Phys. Rev. B 51 10436; Antonov V N, Oppeneer P M, Yaresko A N, Perlov A Ya and Kraft T 1997 Phys. Rev. B 56 13012; Galanakis I, Ostanin S, Alouani M, Dreyssé H and Wills J M (2000) Phys. Rev. B 61 4093

[10] Galanakis I, Dederichs P H and Papanikolaou N 2002 Phys. Rev. B 66134428

[11] Nanda B R K and Dasgupta S 2003 J. Phys.: Condens. Matter 157307

[12] Block T, Carey M J, Gurney D A and Jepsen O 2004 Phys. Rev. B 70205114

[13] Orgassa D, Fujiwara H, Schulthess T C and Butler W H 1999 Phys. Rev. B 6013237

[14] Larson P, Mahanti S D and Kanatzidis M G 2000 Phys. Rev. B 6212754

[15] Kirillova M N, Makhnev A A, Shreder E I, Dyakina V P and Gorina N B 1995 Phys. Status Solidi (b) 187231

[16] Hanssen K E H M and Mijnarends P E 1990 Phys. Rev. B 34 5009; Hanssen K E H M, Mijnarends P E, Rabou L P L M and K.H.J. Buschow K H J 1990 Phys. Rev. B 421533

[17] Clowes S K, Miyoshi Y, Bugoslavsky Y, Branford W R, Grigorescu C, Manea S A, Monnereau O and Cohen L F 2004 Phys. Rev. B 69214425

[18] van Roy W, de Boeck J, Brijs B and Borghs G 2000 Appl. Phys. Lett. 77 4190; van Roy W, Borghs G and de Boeck J 2001 J. Cryst. Growth 227-228 862; van Roy W, Borghs G and de Boeck J 2002 J. Magn. Magn. Mater. 242 489; van Roy W, Wojcik M, Jedryka E, Nadolski S, Jalabert D, Brijs B, Borghs G and de Boeck J 2003 Appl. Phys. Lett. 83 4214; Wojcik M, van Roy W, Jedryka E, Nadolski S, Borghs G and de Boeck J 2002 J. Magn. Magn. Mater. 240414

[19] Gardelis S, Androulakis J, Giapintzakis J, Monnereau O and Buckle P D 2004 Appl. Phys. Lett. 85 3178; Gardelis S, Androulakis J, Migiakis P, Giapintzakis J, Clowes S K, Bugoslavsky Y, Branford W R, Miyoshi Y and Cohen L F 2004 J. Appl. Phys. 95 8063; Giapintzakis J, Grigorescu C, Klini A, Manousaki A, Zorba V, Androulakis J, Viskadourakis Z and Fotakis C 2002 Appl. Surf. Sci. 197 421; Giapintzakis J, Grigorescu C, Klini A, Manousaki A, Zorba V, Androulakis J, Viskadourakis Z and Fotakis C 2002 Appl. Phys. Lett. 80 2716; Grigorescu C E A, Manea S A, Mitrea M, Monnereau O, Rotonier R, Tortet L, Keschawarz R, Giapintzakis J, Klini A, Zorba V, Androulakis J and Fotakis C 2003 Appl. Surf. Sci. 21278

[20] Bach P, Rüster C, Gould C, Becker C R, Schmidt G and Molenkamp L W 2003 J. Cryst. Growth 251 323; Bach P, Bader A S , Rüster C, Gould C, Becker C R, Schmidt G, Molenkamp L W, Weigand W, Kumpf C, Umbach E, Urban R, Woltersdorf G and Heinrich B 2003 Appl. Phys. Lett. 83521

[21] Soulen Jr R J, Byers J M, Osofsky M S, Nadgorny B, Ambrose T, Cheng S F, Broussard P R, Tanaka C T, Nowak J, Moodera J S, Barry A and Coey J M D 1998 Science 28285

[22] Mancoff F B, Clemens B M, Singley E J and Basov D N 1999 Phys. Rev. B 60 R12565; Zhu W, Sinkovic B, Vescovo E, Tanaka C and Moodera J S 2001 Phys. Rev. B 64 R060403; Bona G L, Meier F, Taborelli M, Bucher E and Schmidt P H 1985 Solid State Commun. 56391

[23] Caballero J A, Reilly A C, Hao Y, Bass J, Pratt W P, Petroff F and Childress 1999 J. Magn. 
Magn. Mater. 198-199 55; Kabani R, Terada M, Roshko A and Moodera J S $1990 \mathrm{~J}$. Appl. Phys. 674898

[24] Ristoiu D, Nozières J P, Borca C N, Komesu T, Jeong H -K and Dowben P A 2000 Europhys. Lett. 49 624; Ristoiu D, Nozières J P, Borca C N, Borca B and Dowben P A 2000 Appl. Phys. Lett. 76 2349; Borca C N, Komesu T, Jeong H -K, Dowben P A, Ristoiu D, Hordequin Ch, Pierre J and Nozières J P 2000 Appl. Phys. Lett. 7788

[25] Galanakis I 2002 J. Phys.: Condens. Matter 146329

[26] Chioncel L, Katsnelson M I, de Groot R A and Lichtenstein A I 2003 Phys. Rev. B 68144425

[27] Skomski R and Dowben P A 2002 Europhys. Lett. 58 544; Dowben P A and Skomski R 2003 J. Appl. Phys. 93 7948; Dowben P A and Skomski R 2004 J. Appl. Phys. 957453

[28] Borca C N, Komesu T, Jeong H -K, Dowben P A, Ristoiu D, Hordequin Ch, Nozières J P, Pierre J, Stadler Sh and Idzerda Y U 2001 Phys. Rev. B 64 052409; Ritchie L, Xiao G, Ji Y, Chen T Y, Chien C L, Zhang M, Chen C, Liu Z, Wu G and Zhang X X 2003 Phys. Rev. B 68104430

[29] Jenkins S J and King D A 2001 Surf. Sci. 494 L793

[30] Jenkins S J 2004 Phys. Rev. B 70245401

[31] Galanakis I 2005 J. Magn. Magn. Mater. 288411

[32] Perdew J P, Burke K and Ernzerhof M 1996 Phys. Rev. Lett. 773865

[33] Wimmer E, Krakauer H, Weinert M and Freeman A J 1981 Phys. Rev. B 24 864; Weinert M, Wimmer E and Freeman A J 1982 Phys. Rev. B 264571

[34] http://www.fleur.de

[35] Otto M J, van Woerden R A M, van der Valk P J, Wijngaard J, van Bruggen C F, Haas C and Buschow K H J 1989 J. Phys.: Condens. Matter 12341

[36] Plogmann S, Schlathölter T, Braun J, Neumann M, Yarmoshenko Yu M, Yablonskikh M V, Shreder E I, Kurmaev E Z, Wrona A and Ślebarski A 1999 Phys. Rev. 606428

[37] Mavropoulos Ph, Sato K, Zeller R, Dederichs P H, Popescu V and Ebert H 2004 Phys. Rev. B 69 054424; Mavropoulos Ph, Galanakis I, Popescu V and Dederichs P H 2004 J. Phys.: Condens. Matter 16 S5759

[38] Galanakis I 2005 Phys. Rev. B 71012413

[39] Borca C N, Komesu T and Dowben P A 2002 J. Electron Spectrosc. Relat. Phenom. 122259 